\title{
Comparative Study and Analysis of Medical Image Fusion Techniques
}

\author{
Pramit Parekh \\ Chandubhai S. Patel \\ Inst. of Technology, \\ Charusat \\ Changa, Anand, \\ Gujarat
}

\author{
Nehal Patel, \\ Robinson \\ Macwan \\ Chandubhai S. Patel \\ Inst. of Technology, \\ Charusat \\ Changa, Anand, \\ Gujarat
}

\author{
Priteshkumar \\ Prajapati \\ Chandubhai S. Patel \\ Inst. of Technology, \\ Charusat \\ Changa, Anand, \\ Gujarat
}

\author{
Sarita Visavalia \\ Chandubhai S. Patel \\ Inst. of Technology, \\ Charusat \\ Changa, Anand, \\ Gujarat
}

\begin{abstract}
The term fusion that means in general an approach of extraction of information acquired from several domains. Basically, Image Fusion can be defines as a process of combining information from multiple input images in such way that final fused image having good quality information then individual image. Medical imaging field demands images with high resolution and higher information contents for necessary disease diagnosis and visualization. Therefore, in practical scenario we required more complementary information for necessary disease diagnosis purpose. In this paper, Image Fusion techniques are broadly classified as spatial domain fusion techniques and Transform domain fusion techniques. Various techniques in each class are discussed in short description. In this study Performance of various techniques are evaluated using quality measures such as Entropy, Peak Signal to Noise Ratio, Signal to Noise Ratio, and Structural Similarity Index. For analysis purpose one can considered CT and MRI images of same scene.
\end{abstract}

\section{General Terms}

Medical image fusion, Image fusion Techniques, Fuzzy Inference System

\section{Keywords}

Multi-Modality, CT (Computed Tomography), MRI (Magnetic Resonance Imaging), MIN-SUM-MOM (Minimum Sum Mean of Maximum), FIS (Fuzzy Inference System)

\section{INTRODUCTION}

Image Fusion is a mechanism to improve the quality of information from a set of images. By the process of image fusion the good information from each of the given images is fused together to form a resultant image whose quality is superior to any of the input images [2]. As we know that medical imaging field demands more complementary information for disease diagnosis purpose. However, this is not possible using single modality medical images as X-ray computed tomography (CT) is suited only for recognizing bones structure, MRI giving clear information about the soft tissues and so on. . In this regard, medical image fusion is the only emerging technique which has attracted researchers to assist the doctors in fusing images and retrieving relevant information from multiple modalities such as CT, MRI, FMRI, SPECT, PET [1].

The rest of sections are organized in this paper as follows: In Section-II Spatial domain fusion techniques are describes in concisely; Section-III deal with different image fusion techniques in Transform domain; In Section-IV We will see shortly how we can used Fuzzy technique i.e. MIN-SUMMOM in medical image fusion; Section-V deal with the some quality measures are used in analysis; In section-VI shows the comparison of different medical image fusion techniques; Section-VII, conclusion of this study is summarized.

\section{SPATIAL DOMAIN FUSION TECHNIQUES}

This class of fusion is directly applied to the input images which decrease the signal-to-noise ratio of the final fused image with the simple averaging technique.

\subsection{Average Method}

Average Method working as averaging every corresponding pixel of input images to obtain final fused image [3]. It is define as:

$$
\mathrm{F}(x, y)=P(x, y)+Q(x, y) / 2
$$

Where, $\mathrm{F}(\mathrm{x}, \mathrm{y})$ is the final fused image, $\mathrm{P}(\mathrm{x}, \mathrm{y})$ and $\mathrm{Q}(\mathrm{x}, \mathrm{y})$ are two input images [3].

\subsection{Select Maximum Method}

In this method, the pixel with maximum intensity from the corresponding spatial locations from all the images to be fused is selected as the resultant pixel of the fused output image. The advantage of this method over averaging method is that there is no compromise made over the good information available in the input images. But the disadvantage is that it considers only the higher pixel intensity as the better information ignoring all other values [4].

\subsection{Select Minimum Method}

This is similar to the select maximum method but with the difference, it considers only the pixel with lowest intensity value and ignores all other values. This method also has the disadvantage of either completely considering information or discarding it fully [4]. 


\subsection{PCA (Principal Component Analysis)}

PCA is one of the linear mapping Techniques used in many application of image processing. It is known as a general statistical method that transforms correlated variables into uncorrelated variables. These new variables are getting as linear combination of the original variables. Principal components are linear combinations of optimally weighted observed variables [2].

In Principal Component Analysis (PCA), extracted components are equivalent to observed variables which are being analyzed. However, in most PCA analysis, only the first few components has the largest possible variance, so only these first few components are retained, interpreted $\&$ used for subsequent analysis.

\subsection{IHS (Intensity Hue Saturation) Fusion Method}

IHS is most widely used perceptual color model in image analysis. IHS transformation is one of the most popular fusion methods used in many application widely used in Remote sensing applications. IHS largely explains the popularity of perceptual colour space and overcomes the limitation of commonly used RGB colour space which does not relate intuitively to the attribute of human colour perception. RGB color space is normally used to represent the range of colors displayable on a computer monitor [5].

Intensity can be defined as total amount of light that reaches the eye. Hue is defined as the predominant wavelength of a color and saturation is the purity of total amount of white light of a color. The principle of the IHS transformation to merge images attributes to the fact that the IHS colour space is supplied to intellectual system of human beings and that the transformation has the ability to separate the spectral information of an RGB composition into its two components $\mathrm{S}$ and $\mathrm{H}$. while the most of the spatial information in the I component [5].

\subsection{Brovey Method}

This method is developed and supported by an American scientist. Brovey method is also known as color normalization transform because it contains a red-green-blue (RGB) color transform method. The Brovey transformation was developed to avoid the drawbacks of the multiplicative method. It is a simple method for combining data from different sensors. In this method combination of arithmetic operation are used and spectral bands are normalized before they are multiplied with the panchromatic image. It also retains the corresponding spectral feature of each pixel and transforms all the luminance information into a panchromatic image of high resolution [9].

The disadvantage of spatial domain fusion is that produce spatial distortion in fused image. This problem can be solved by transform domain fusion.

\section{TRANSFORM DOMAIN FUSION TECHNIQUES}

In transform domain fusion firstly the input images are decomposed based on transform coefficients. Then the fusion technique is applied and the fusion decision map is obtained. Inverse transformation on that decision map produce fused image. Transform domain fusion enables the image's salient features are more clearly visible than in spatial domain fusion. Transform domain fusion techniques are outlined as follows:

\subsection{DCT (Discrete Cosine Transform)}

In this method, images which are fused, firstly divided in to non-Overlapping block having size $\mathrm{NxN}$. DCT coefficients are computed for each block and fusion rules are applied to get fused DCT coefficients. IDCT is then applied on the fused coefficients to produce the fused image/block.

\subsection{Pyramid Method}

Pyramid method consists of set of lowpass or bandpass copy of an image. Each copy is representing pattern information of a different scale. Basically, in pyramid method every level is a factor of two smaller as its predecessor, and higher level is focus on lower partial frequencies. This pyramid does not contain all information regarding to reconstruct the original image [10].

\subsection{DWT (Discrete Wavelet Transform)}

In discrete wavelet transform filters are specially design so that successive layer of pyramid only contains details which are not available on preceding levels.

The DWT decomposition uses a cascade of special low-pass and high-pass filters and a sub-sampling operation. 2D-DWT generates four images as output that having size which is equal to half of original image. So from first input image we will get $\mathrm{HHa}$, HLa, LHa, LLa images and from second input image we will get $\mathrm{HHb}, \mathrm{HLb}, \mathrm{LHb}$, LLb images. LH means that low-pass filter is applied along $\mathrm{x}$ and followed by high pass filter along $y$. The LL image contains the approximation coefficients. LH image contains the horizontal detail coefficients. HL image contains the vertical detail coefficients; HH contains the diagonal detail coefficients. The wavelet transform can be performed for multiple levels. The next level of decomposition is performed using only the LL image. The result is four sub-images each of size equal to half the LL image size [2].

\subsection{RDWT (Redundancy DWT)}

Redundancy discrete wavelet transform (RDWT) is another variant of wavelet transform, is used to overcome the shift variance problem of DWT. RDWT have been applied in various image processing application but not well researched in medical image fusion research area. RDWT can be considered as an approximation to DWT that removes the down-sampling operation from traditional critically sampled DWT, produces an over-complete representation and provides noise per-sub-band relationship. The shift variant characteristic of DWT arises from the use of down- sampling whereas RDWT is shift invariant because the spatial sampling rate is fixed across scale [1] [6].

\subsection{SWT (Stationary Wavelet Transform)}

Stationary Wavelet Transform (SWT) is similar to Discrete Wavelet Transform (DWT) but the only process of down-sampling is suppressed that means the SWT is translation-invariant.

The 2D Stationary Wavelet Transform is based on the idea of no decimation. It uses the DWT and eliminates both down sampling in forward transform and up sampling in inverse transform. More specifically, it applies the transform at each point of the input image and saves the detail coefficients and then uses the low frequency information at each level [7].

\section{IMAGE FUSION USING MIN-SUM- MOM}

Mamdani type minimum-sum-mean of maximum is pixel level image fusion technique. This technique is implemented 
in three steps. First step is about to create Fuzzy sets that is used for describing different gray levels of input images and building the fuzzy inference system (FIS). In second step, fuzzy inference is carried out according to the fuzzy rules and the membership degree of each output pixel is obtained. Finally, in third step, the output gray level value is calculated through defuzzification and the fused image is obtained [1].

\section{QUALITY MEASURES IN PERFORMANCE EVALUATION}

Performance evaluation is necessary for determining the result of any image fusion technique in terms of qualitative and quantitative analysis. The following section explains the quality metrics used in analysis of image fusion techniques.

\subsection{Entropy}

Entropy is one of the most important quantitative measures in image fusion. A digital image consists of pixels arranged in rows and columns. Each pixel in Image is defined by its position and gray scale level. For an image consists of L gray levels, the entropy is defined as [8]:

$$
H=-\sum_{i=1}^{L}, P(i) \log 2
$$

Where, $\mathrm{P}(\mathrm{i})$ is probability of each grey scale level.

\subsection{SNR (Signal to Noise Ratio)}

SNR is defined as the ratio of mean pixel value to standard deviation of the corresponding pixel values. It is define as:

$$
\text { SNR = Mean } / \text { Standard deviation }
$$

$$
\text { (3) }
$$

SNR provides Contrast Information that image contains. Higher value of the SNR indicates that image having more contrast.

\subsection{PSNR (Peak Signal to Noise Ratio)}

PSNR is used to measure quality of input image with respect to the input image. It is define as:

$$
\begin{aligned}
& M S E=1 / \mathrm{pq} \sum_{i=0}^{r-1} * \sum_{i=0}^{s-1}[A(i, j)-B(i, j)]^{2} \\
& P S N R=10 \log _{10}\left(M A X^{2} \mid M S E\right)
\end{aligned}
$$

Where, MAX is the maximum value in an image. $p, q$ are the height and weight of an image. A(i,j) is the value of input image and $B(i, j)$ is the value of fused image [1].

\subsection{SSIM (Structural Similarity Index)}

SSIM provides the association between the changes of structural information within the images and the perceived distortion of the images. It can be defined as a measure to evaluate similarity between two images $\mathrm{X}$ and $\mathrm{Y}$ by the following expression [1].

$$
\begin{aligned}
& \operatorname{SSIM}(X, Y)=\left(\left(2\left(\mu_{X} \mu_{Y}+k 1\right)\right) * 2\left(\sigma_{X Y}+k 2\right)\right) / \\
& \left(\left(\mu_{X}^{2}+\mu_{Y}^{2}+k 1\right) *\left(\sigma_{X}^{2}+\sigma_{Y}^{2}+k 2\right)\right)
\end{aligned}
$$

Where, $\mu_{\mathrm{X}}$ and $\mu_{\mathrm{Y}}$ denotes the mean intensities $\sigma_{\mathrm{X}}$ and $\sigma_{\mathrm{Y}}$ denote the standard deviation, $\sigma_{X Y}$ gives the covariance of $X$ and $\mathrm{Y}$, and $\mathrm{k} 1$ and $\mathrm{k} 2$ are constants.

\section{COMPARISON OF MEDICAL IMAGE FUSION TECHNIQUES}

For Analysis purpose in this study two multimodal medical images CT and MRI of the single person are taken as an input.

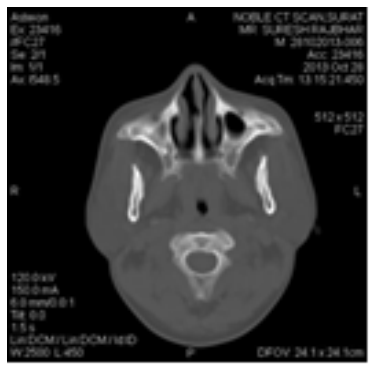

CT

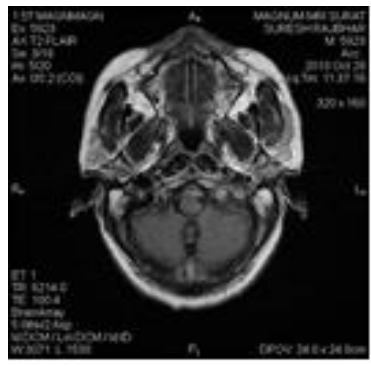

MRI
Fig 1: Original Multimodal medical image dataset

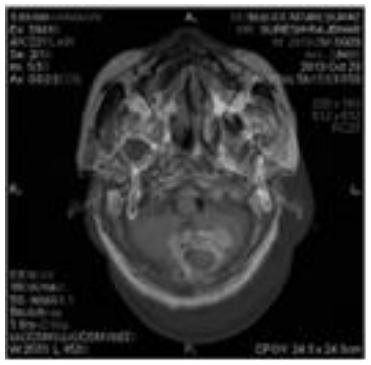

Average

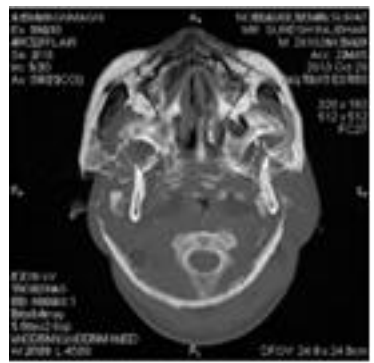

Maximum
Fig 2: Fused images using Average and Maximum

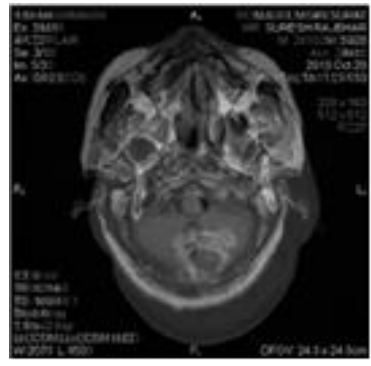

PCA

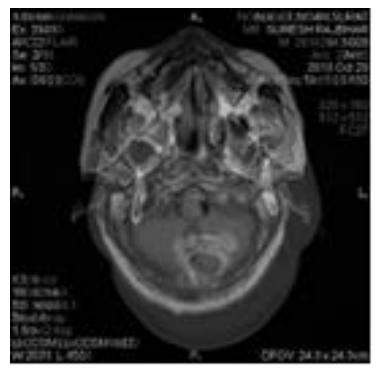

Laplacian Pyramid
Fig 3: Fused images using PCA and Laplacian Pyramid

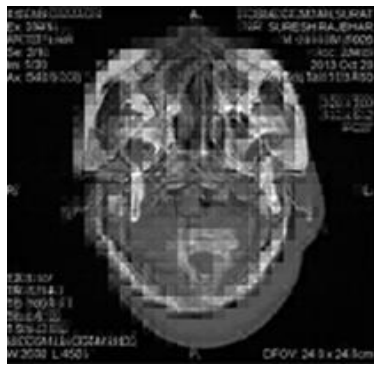

DWT

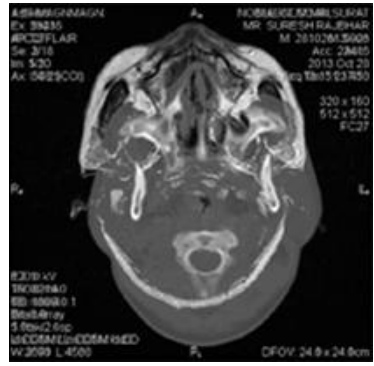

MIN-SUM-MOM
Fig 4: Fused images using DWT and MIN-SUM-MOM 
Table 1. Quantitative Analysis

\begin{tabular}{|c|c|c|c|c|c|c|c|}
\hline \multirow{4}{*}{$\begin{array}{c}\text { Input } \\
\text { Images }\end{array}$} & \multirow{2}{*}{ Parameters } & \multicolumn{7}{|c|}{ Techniques } \\
\cline { 3 - 8 } & & Maximum & Averaging & PCA & $\begin{array}{c}\text { Laplacian } \\
\text { Pyramid }\end{array}$ & DWT & MIN-SUM-MOM \\
\hline \multirow{4}{*}{ C1.jpg } & PSNR & 31.9243 & 33.6611 & 33.4303 & 33.6611 & 49.0438 & 93.2853 \\
\cline { 2 - 8 } & SSIM & 33.2678 & 20.4436 & 18.8690 & 20.4436 & 18.1178 & 31.1589 \\
\cline { 2 - 9 } & SNR & 0.9614 & 0.9222 & 0.9198 & 0.9222 & 0.9484 & 0.9667 \\
\cline { 2 - 9 } & ENTROPY & 0.2932 & 0.4485 & 0.4717 & 0.4485 & 0.5824 & 5.1286 \\
\cline { 2 - 8 } M1.jpg & PSNR & 32.4152 & 33.6611 & 33.9047 & 33.6611 & 49.0535 & 86.7532 \\
\cline { 2 - 8 } & SSIM & 58.2547 & 37.6462 & 41.3375 & 37.6462 & 31.5243 & 49.4951 \\
\hline
\end{tabular}

In above Table 1, (C1.jpg) is a CT image and (M1.jpg) is a MRI image of single patient. Input images of CT and MRI are considered as one group. One can computed different quality measures for various Medical Image Fusion Techniques as listed in Table 1. All images having $256 * 256$ pixels. In Figure 1 show the sample set of image that is Dataset 1. This Dataset is taken from the Noble CT-Scan, Surat. For Analysis purpose 6 groups of multimodal medical images are taken.

Implementations of Medical image fusion with different techniques have been done using MATLAB Tool.

Now, following figures displays the graphs of different 6 datasets with the different quality measures. Here, in this study only few techniques for analysis PCA, Laplacian Pyramid, DWT, and MIN-SUM-MOM are taken.

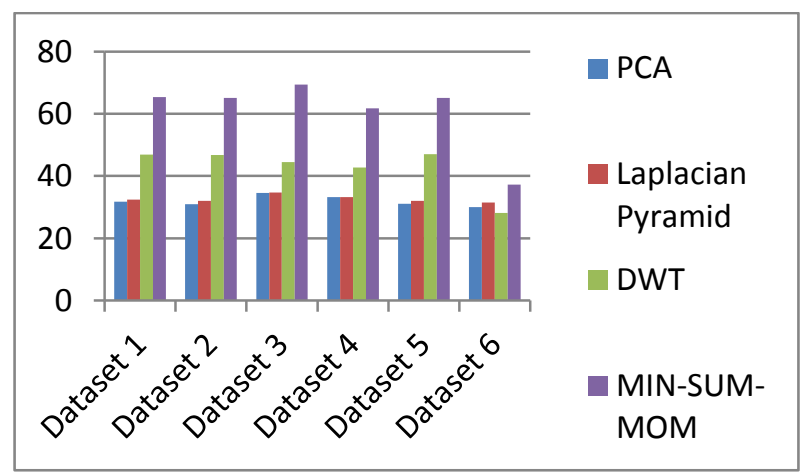

Fig 5: Graph-1 PSNR Calculation with MRI images

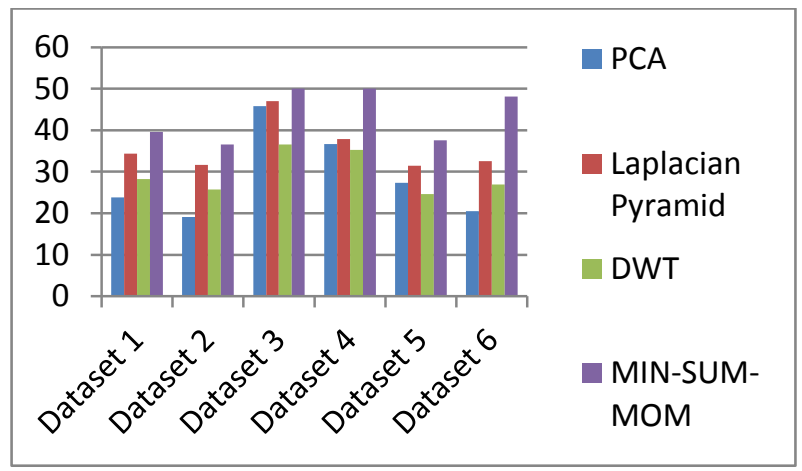

Fig 6: Graph-2 SSIM Calculation with MRI image

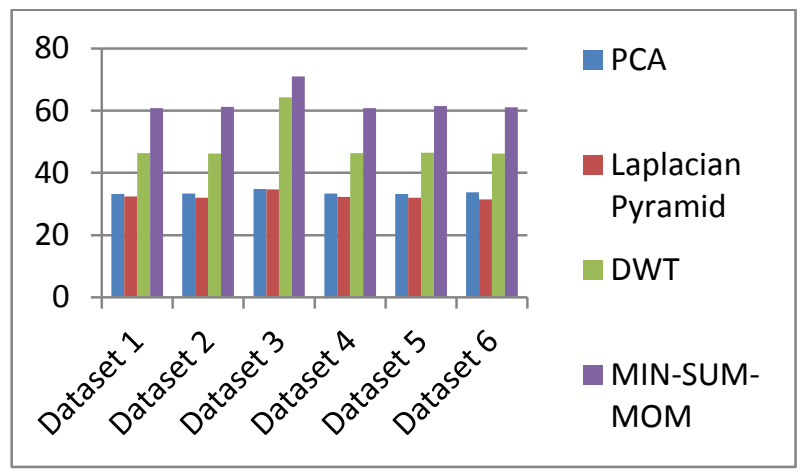

Fig 7: Graph-3 PSNR Calculation with CT images

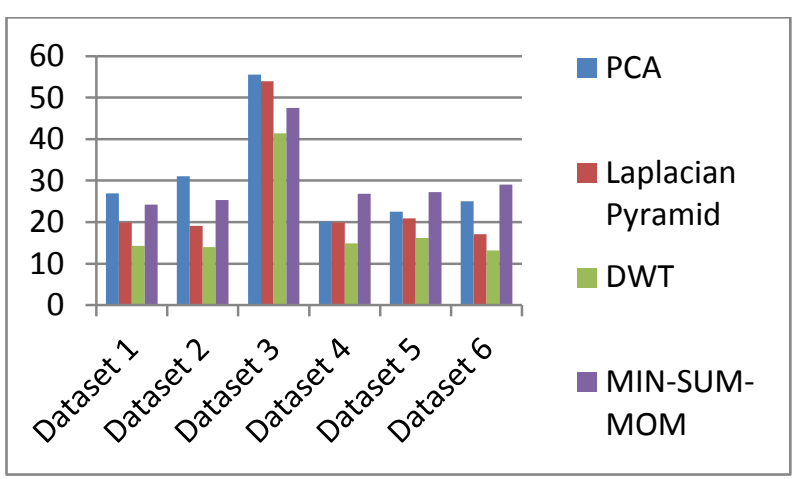

Fig 8: Graph-4 SSIM Calculation with CT images

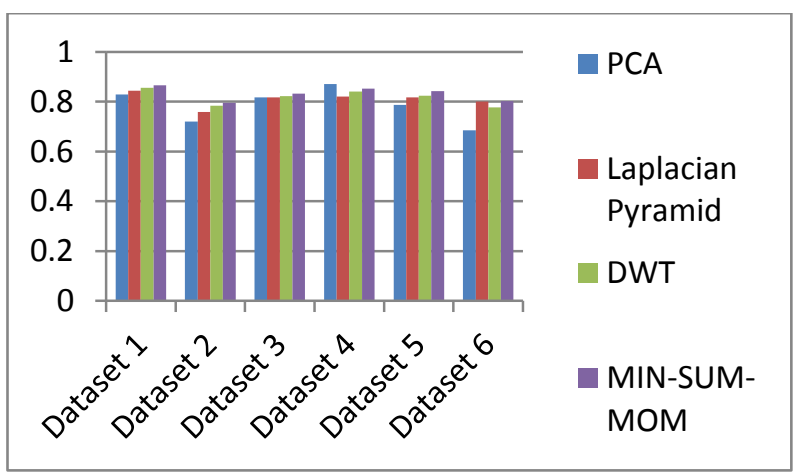

Fig 9: Graph-5 SNR Calculation 


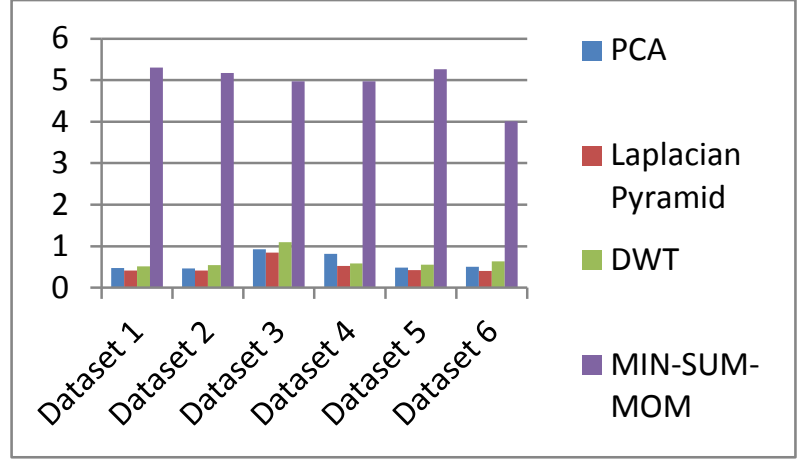

Fig 10: Graph-6 Entropy Calculation

\section{CONCLUSION}

In this paper, different medical image fusion techniques in both spatial and transform domain have been studied. Each technique having its own Pros and Cons depends on the application. In medical imaging field, demands more information content and visualization in an image. Therefore, many medical image fusion techniques have been proposed but, still there having certain limitation.

The study of different techniques of medical image fusion shows that FIS type MIN-SUM-MOM gives better performance in visualization perspectives then all other techniques and also more satisfactory fusion result as verified with the quantitative measures.

\section{ACKNOWLEDGMENTS}

The authors would like to thank Noble CT-scan, Surat for providing brain images of same patient at different modality and also thanks to Dr. Milind Sule (Radiologist) of Noble CTScan for giving better idea regarding medical images.

\section{REFERENCES}

[1] Chandra Prakash, S Rajkumar, P.V.S.S.R. Chandra Mouli, "Medical Image Fusion Based on Redundancy DWT and Mamdani Type Min-sum Mean-of-max Techniques with Quantitative Analysis" International Conference on Recent Advances in Computing and Software Systems (2012).
[2] Mr. Rajenda Pandit Desale, Prof. Sarita V. Verma, "Study and Analysis of PCA, DCT \& DWT based Image Fusion Techniques", International Conference on Signal Processing, Image Processing and Pattern Recognition [ICSIPR] (2013).

[3] Prof. Keyur N. Brahmbhatt, Dr. Ramji M. Makwana, "Comparative study on image fusion methods in spatial domain", International journal of advanced research in engineering and technology (IJARET) (2013).

[4] Sruthy S, Dr. Latha Parameswaran, Ajeesh P Sasi, "Image Fusion Technique using DT-CWT", International Multi-Conference on Automation, Computing, Communication, Control and Compressed Sensing (iMac4s) (2013).

[5] Kavita Tewari, Leena Shah, "Pixel Level Image Fusion Based on Spatial and Transform Domain", International Conference on Computer Science, Information and Technology, Pune, ISBN-978-93-81693-83-4 (2012)

[6] S.Rajkumar, S.Kavitha, "Redundancy Discrete Wavelet Transform and Contourlet Transform for Multimodality Medical Image Fusion with Quantitative Analysis" , 3rd International Conference on Emerging Trends in Engineering and Technology (2010).

[7] Mirajkar Pradnya P., Ruikar Sachin D., "Wavelet based Image Fusion Techniques", International Conference on Intelligent Systems and Signal Processing (ISSP) (2013).

[8] M.A. Mohamed, R.M EI-Den, "Implementation of Image Fusion Techniques for Multi-Focus Images Using FPGA”, 28th National radio science conference (2011).

[9] Rohan Ashok Mandhare, Pragati Upadhyay, Sudha Gupta, "Pixel-level image fusion using brovey transforme and wavelet transform", International Journal of Advanced Research in Electrical, Electronics and Instrumentation Engineering(IJAREEIE), Vol. 2, Issue 6, June 2013.

[10] Firooz Sadjadi, "Comparative Image Fusion Analysais", Proceedings of the 2005 IEEE Computer Society Conference on Computer Vision and Pattern Recognition (CVPR'05). 